\title{
Primary Care Providers' Acceptance of Pharmacists' Recommendations to Support Optimal Medication Management for Patients with Diabetic Kidney Disease
}

\author{
Leah L. Zullig, $P h D^{1,2}$, Shelley A. Jazowski, MPH ${ }^{2,3}$, Clemontina A. Davenport, PhD4, \\ Clarissa J. Diamantidis, MD, MHS ${ }^{2,5}$, Megan M. Oakes, MPA', \\ Sejal Patel, BPharm, BCGP5, Jivan Moaddeb, PharmD ${ }^{5,6}$, and Hayden B. Bosworth, \\ $P h D^{7,2,5,7,8}$
}

\begin{abstract}
'Center of Innovation to Accelerate Discovery and Practice Transformation (ADAPD), Durham Veterans Affairs Health Care System, Durham, NC, USA; ${ }^{2}$ Department of Population Health Sciences, Duke University, Durham, NC, USA; ${ }^{3}$ Department of Health Policy and Management, University of North Carolina at Chapel Hill, Chapel Hill, NC, USA; ${ }^{4}$ Department of Biostatistics and Bioinformatics, Duke University, Durham, NC, USA; ${ }^{5}$ Division of General Internal Medicine, Duke University, Durham, NC, USA; ${ }^{\circ}$ Duke Center for Applied Genomics \& Precision Medicine, Duke University, Durham, NC, USA; ${ }^{7}$ School of Nursing, Duke University, Durham, NC, USA; ${ }^{8}$ Department of Psychiatry and Behavioral Sciences, Duke University, Durham, NC, USA.
\end{abstract}

BACKGROUND: Patients with diabetic kidney disease (DKD) often struggle with blood pressure control. In team-based models of care, pharmacists and primary care providers (PCPs) play important roles in supporting patients' blood pressure management.

OBJECTIVE: To describe whether PCPs' acceptance of pharmacists' recommendations impacts systolic blood pressure (SBP) at 36 months.

DESIGN: An observational analysis of a subset of participants randomized to the intervention arm of the Simultaneous risk factor control using Telehealth to slOw Progression of Diabetic Kidney Disease (STOP-DKD) study.

PARTICIPANTS: STOP-DKD participants for whom (1) the pharmacist made at least one recommendation to the PCP; (2) there were available data regarding the PCP's corresponding action; and (3) there were SBP measurements at baseline and 36 months.

INTERVENTION: Participants received monthly telephone calls with a pharmacist addressing health behaviors and medication management. Pharmacists made medication-related recommendations to PCPs.

MAIN MEASURES: We fit an unadjusted generalized linear mixed model to assess the association between the number of pharmacists' recommendations for DKD and blood pressure management and PCPs' acceptance of such recommendations. We used a linear regression model to evaluate the association between PCP acceptance and SBP at 36 months, adjusted for baseline SBP.

KEY RESULTS: Pharmacists made 176 treatment recommendations (among 59 participants), of which 107 (61\%) were accepted by PCPs. SBP significantly declined by an average of $10.5 \mathrm{mmHg}(p<0.01$ ) among 47 of 59 participants who had valid measurements at baseline and 36 months. There was a significant association between the number of pharmacist recommendations and the odds of PCP acceptance (OR 1.19; 95\%CI 1.00, 1.42; $p<0.05$ ),

Received July 12, 2019

Revised August 29, 2019

Accepted September 20, 2019

Published online October 28, 2019 but no association between the number of accepted recommendations and SBP.

CONCLUSIONS: Pharmacists provided actionable medication-related recommendations. We identified a significant decline in SBP at 36 months, but this reduction was not associated with recommendation acceptance.

\section{TRIAL REGISTRATION: NCT01829256}

KEY WORDS: diabetic kidney disease; blood pressure control; team-based primary care; medication management.

J Gen Intern Med 35(1):63-9

DOI: $10.1007 / \mathrm{s} 11606-019-05403-\mathrm{x}$

(c) Society of General Internal Medicine (This is a U.S. government work and not under copyright protection in the U.S.; foreign copyright protection may apply) 2019

\section{INTRODUCTION}

Diabetic kidney disease (DKD) is one of the most prevalent diabetes-related complications, ${ }^{1}$ affecting more than $30 \%$ and $40 \%$ of patients with type I and type II diabetes, respectively. ${ }^{2-4}$ Diagnosis and severity of DKD is associated with an increased risk of adverse health outcomes (e.g., end-stage renal disease, cardiovascular disease/events, end-organ damage), impaired quality of life, and premature mortality. ${ }^{2-4}$ To manage DKD and prevent disease progression, treatment often focuses on controlling a patient's glucose and common comorbidities, including elevated blood pressure. ${ }^{2,5,6}$

Patients experiencing this trifecta of chronic conditions - diabetes, kidney disease, and hypertension - may have competing health demands and especially struggle to manage their blood pressure. Preventing DKD progression and addressing elevated blood pressure requires patients to engage in complex self-management, often comprised of medication intensification, medication management, medication adherence, and concurrently changing multiple health behaviors (e.g., improving diet, increasing physical activity, maintaining 
appropriate self-monitoring). ${ }^{7}$ This process is challenging and may best be approached with a patient-centered, team-based model of care.

Team-based care for DKD may necessitate involvement from patients' primary care providers (PCPs), nephrology care team, and pharmacists, among others. Pharmacists may play a particularly critical role in the context of managing concurrent DKD and elevated blood pressure, because of their unique perspectives on medications prescribed across conditions. Evidence supports the integration of pharmacists as part of the ambulatory care team for the treatment of patients with DKD. Specifically, physician-pharmacist team-based care has been associated with a decline in the incidence of end-stage renal disease $^{8}$ and improvements in both medication adherence ${ }^{9}$ and health outcomes. ${ }^{10,11}$ Moreover, pharmacist-delivered interventions appear to be successful at enhancing adherence and subsequent clinical outcomes even when delivered remotely over the telephone. ${ }^{12-16}$

We recently completed the Simultaneous risk factor control using Telehealth to slOw Progression of Diabetic Kidney Disease (STOP-DKD) study (trial registration: NCT01829256). Details of the STOP-DKD approach have been previously described elsewhere. ${ }^{17}$ STOP-DKD provided an innovative care delivery model, where pharmacists recommended medication-related changes to PCPs of patients with DKD. Since the central premise of STOP-DKD required communication-informed action between PCPs and pharmacists, the purpose of this analysis was to describe whether PCPs' acceptance of pharmacists' recommendations had an impact on systolic blood pressure (SBP) decline at 36 months.

\section{METHODS}

\section{Study Design and Setting}

The STOP-DKD study was a two-arm randomized controlled trial that evaluated the effectiveness of a multifactorial behavioral and medication management intervention to reduce kidney function decline at three years compared with usual care. Patients with DKD were recruited from seven primary care clinics affiliated with the Duke University Health System and located in North Carolina. STOP-DKD required a team-based approach to healthcare delivery, whereby PCPs fulfilled their usual care roles and research pharmacists interacted with study participants over the telephone for 36 months, delivering monthly behavioral-educational modules focused on home blood pressure monitoring, medication management, and diabetes self-management. ${ }^{17}$ When clinically indicated, the two research pharmacists electronically made treatment recommendations to a participant's PCP based on evidence-based protocols. ${ }^{17}$ These recommendations were based on participants' home-based blood pressure values. Examples of research pharmacist recommendations are reported in Table 1.

Study assessments were conducted at baseline enrollment, 12 months, 24 months, and 36 months. At each assessment,
Table 1 Example Research Pharmacist Recommendations

\begin{tabular}{ll}
\hline \hline $\begin{array}{l}\text { Type of } \\
\text { recommendation }\end{array}$ & Example of recommendation \\
\hline Appropriateness & $\begin{array}{l}\text { • Patient should re-start carvedilol-Rx was not } \\
\text { refilled by PCP so patient believed it was } \\
\text { discontinued. }\end{array}$ \\
Intensification & $\begin{array}{l}\text { Patient should have BP medication } \\
\text { intensified. }\end{array}$ \\
& $\begin{array}{l}\text { However, most recent value }<135 / 85 . \\
\text { Re-check BP at office visit. If }>140 / 90, \\
\text { intensify regimen. } \\
\text { - BP }>135 / 85 . \text { On max dose of lisinopril. } \\
\text { Consider adding low dose of HCTZ or } \\
\text { chlorthalidone. } \\
\text { - Patient has had episodes of hypoglycemia. } \\
\text { Did not reduce glimepiride and took higher } \\
\text { dose of Lantus. }\end{array}$ \\
\hline
\end{tabular}

$B P$ blood pressure, HCTZ hydrochlorothiazide, PCP primary care provider

demographic, social, and clinical data were collected. Research staff collected three sets of blood pressure values (SBP, diastolic blood pressure, and mean arterial pressure) using Omron's Professional Digital Blood Pressure Monitor (Model HEM-907XL), following a standardized protocol. ${ }^{17}$ This protocol specified that patients should rest for five minutes prior to blood pressure measurement.

\section{Participants}

The parent STOP-DKD trial randomized 281 participants, of which 138 were allocated to the intervention arm. ${ }^{17}$ Previous reports of STOP-DKD have described participants' baseline characteristics and information regarding participants' ambulatory blood pressure measurements. ${ }^{17,18}$ Our analysis was limited to participants for whom (1) the research pharmacist made at least one clinical recommendation to the PCP; (2) there were available data regarding the PCP's corresponding action; and (3) there were valid SBP measurements at both baseline and 36 months.

\section{Key Measures}

There were two main outcome measures. The first was a binary indicator of a PCP's acceptance of the research pharmacist's recommendations for supporting patients' DKD and blood pressure management. Acceptance was defined as whether or not a PCP acknowledged and took action based on a research pharmacist's recommendation(s) for care. The second key outcome was SBP at 36 months.

\section{Statistical Analysis}

We conducted statistical analysis in SAS version 9.4 (Cary, NC) and R version 3.4.4 (Vienna, Austria). We computed descriptive statistics using means and standard deviations and medians and interquartile ranges (25th to 75 th percentiles) for continuous variables and frequencies and percentages for categorical variables. We performed additional exploratory 
analyses to assess the distributions of SBP values via histograms, density plots, and boxplots and assessed pairwise correlations between continuous variables.

To assess a provider's acceptance of the research pharmacist's treatment recommendation, we fit a generalized linear mixed model (GLMM) to account for the correlation between multiple recommendations made on behalf of the same participant. The number of recommendations was aggregated over the study period for each study participant and was modeled as a fixed effect to address whether a recommendation was the first, second, third, etc. for a particular participant. Each recommendation was treated individually and duplication of recommendation content was not captured. In other words, in the mixed model, the first recommendation would have a value of 0 (not accepted) and the second recommendation would have a value of 1 (accepted). Lastly, we included a random intercept for each study participant, but did not include a random effect for each research pharmacist as $94 \%$ of recommendations originated from one pharmacist.

To evaluate the association between PCP acceptance and SBP at 36 months, we fit two separate linear regression models. In the first model, we regressed SBP on the total number of pharmacist recommendations, the total number of recommendations accepted by the PCP, and baseline SBP. The second model only adjusted for the percent of recommendations accepted by the PCP (defined as the total number of accepted recommendations/the total number of made recommendations) and baseline SBP. This study was approved by the Duke Health Institutional Review Board.

\section{RESULTS}

Participant Characteristics. During the 36-month study period, the research pharmacists made 176 treatment recommendations. These recommendations were made for approximately $43 \%$ of STOP-DKD intervention participants $(n=59)$. Among those participants that received a recommendation, the median age at study enrollment was 63.04 years (interquartile range [IQR] 57.85, 68.11). Most participants were black $(51 \%)$ and male $(61 \%)$. The median SBP at baseline was $139 \mathrm{mmHg}$ (IQR $132.83 \mathrm{mmHg}, 149.50 \mathrm{mmHg}$ ). Additional information regarding participant characteristics and pharmacist recommendations are reported in Table 2.

Pharmacist Interactions. Overall, the mean phone call length across all completed encounters was approximately 17.5 minutes (standard deviation [SD] 8.8 minutes). There was approximately a five-minute average variation in call length by pharmacist. The two pharmacists had average phone calls of approximately 13.2 minutes (SD 8.6 minutes) and 18.2 minutes (SD 8.6 minutes), respectively.

Of the 176 recommendations made, approximately $32 \%$ ( $n$ $=56)$ addressed appropriateness of therapy, $55 \%(n=96)$ addressed intensification, and 14\% $(n=24)$ addressed safety. An example of an appropriateness-related recommendation was as follows: "Stopped spironolactone 3 months ago. Recommended she contact PCP about when/if to re-start." An example of an intensification recommendation was as follows: "Wrist monitor. BP $>135 / 85$. Intensify if office value > 140/90. Consider HCTZ 12.5 mg." An example of a safety recommendation was as follows: "Patient having frequent hypoglycemic readings, recommended to $\mathrm{D} / \mathrm{C}$ sulfonylurea."

Provider Acceptance. Of the 59 participants included in this analysis, approximately one-third of participants $(n=19)$ had only one pharmacist recommendation, while $20 \%(n=12)$ of participants received two medication-related recommendations. For approximately $17 \%$ of participants $(n=10)$, none of the pharmacist recommendations were accepted by their PCP. In the majority of cases, these recommendations addressed intensification. Overall, 61\% $(n=107)$ of all recommendations were accepted by PCPs. There was an interpharmacist difference with $82 \%$ and $59 \%$ of each pharmacist's recommendations being accepted by PCPs. There was a significant correlation between the number of treatment recommendations made by pharmacists and the number of recommendations accepted by PCPs (Pearson's $\rho=0.75, p<0.01$ ).

In the GLMM, there was a significant association $(p<0.05)$ between the number of pharmacist recommendations and the odds of PCPs' acceptance (odds ratio [OR] 1.19, 95\% confidence interval $[\mathrm{CI}] 1.00,1.42$ ) (Table 3 ).

Change in Blood Pressure. Of the 59 participants for whom a treatment recommendation was made, 47 participants $(80 \%)$ had sufficient baseline and follow-up SBP data available for further analysis (Fig. 1). Over the three-year study period, SBP significantly declined on average, by $10.5 \mathrm{mmHg}(p<0.01)$ (median decline $9 \mathrm{mmHg}, p<0.01$ ). The number of recommendations accepted by PCPs and improvement in SBP were positively, but not significantly, correlated (Pearson's $\rho=0.20, p=0.19$ ).

In the first linear regression model, there was no significant association between either the number of recommendations or the number of accepted recommendations and SBP at 36 months $(-0.21,95 \% \mathrm{CI}-3.72,3.30$ and $-2.43,95 \% \mathrm{CI}-$ $7.77,2.90$, respectively). Similarly, there was no association between the percent of accepted recommendations and SBP at 36 months $(2.36,95 \% \mathrm{CI}-12.93,17.63)$ in the second linear regression model (Table 3 ).

\section{DISCUSSION}

The population of patients with DKD is on the rise. ${ }^{19,20}$ Patients with DKD are at increased risk of uncontrolled blood pressure and subsequent health complications, ${ }^{2-4}$ making this an important patient population with complex self- 
Table 2 Baseline Characteristics of Participants with at Least One Pharmacist Recommendation

\begin{tabular}{|c|c|c|c|}
\hline & $\begin{array}{l}\text { Participants } \\
(N=59)\end{array}$ & $\begin{array}{l}\text { No recommendations accepted by PCP } \\
(N=10)\end{array}$ & $\begin{array}{l}\text { At least } 1 \text { recommendation accepted by } \\
\text { PCP }(N=49)\end{array}$ \\
\hline \multicolumn{4}{|l|}{ Demographics } \\
\hline Age (mean, SD) & $61.63(8.97)$ & $64.14(5.92)$ & $61.12(9.44)$ \\
\hline Age (median, IQR) & $63.04(57.85-68.11)$ & $63.28(62.27-67.6)$ & $62.58(57.46-67.69)$ \\
\hline \multicolumn{4}{|l|}{$\operatorname{Sex}(N, \%)$} \\
\hline Female & $23(38.98)$ & $5(50.00)$ & $18(36.73)$ \\
\hline Male & $36(61.02)$ & $5(50.00)$ & $31(63.27)$ \\
\hline \multicolumn{4}{|l|}{ Race $(N, \%)$} \\
\hline White or Caucasian & $25(42.37)$ & $5(50.00)$ & $20(40.82)$ \\
\hline Black/African American & $30(50.85)$ & $5(50.00)$ & $25(51.02)$ \\
\hline Asian & $1(1.69)$ & $0(0.00)$ & $1(2.04)$ \\
\hline Other & $3(5.08)$ & $0(0.00)$ & $3(6.12)$ \\
\hline \multicolumn{4}{|l|}{ Household income $(N, \%)$} \\
\hline$<\$ 15,000$ & $8(13.56)$ & $3(30.00)$ & $5(10.20)$ \\
\hline$\$ 15,000-\$ 29,999$ & $4(6.78)$ & $0(0.00)$ & $4(8.16)$ \\
\hline$\$ 30,000-\$ 59,999$ & $20(33.90)$ & $3(30.00)$ & $17(34.69)$ \\
\hline$\$ 60,000-\$ 89,999$ & $16(27.12)$ & $1(10.00)$ & $15(30.61)$ \\
\hline$\geq \$ 90,000$ & $10(16.95)$ & $2(20.00)$ & $8(16.33)$ \\
\hline Don’t know & $1(1.69)$ & $1(10.00)$ & $0(0.00)$ \\
\hline \multicolumn{4}{|l|}{ Clinical characteristics } \\
\hline \multicolumn{4}{|l|}{ Blood pressure measures } \\
\hline Baseline SBP (mean, SD) & $140.57(14.75)$ & $134.30(9.48)$ & $141.85(15.37)$ \\
\hline Baseline SBP (median, & $139.00(132.83-$ & $133.67(129.92-136.75)$ & $141.33(133.33-150.00)$ \\
\hline IQR) & $149.50)$ & & \\
\hline 36 M SBP (mean, SD) & $129.95(20.90)$ & $126.59(19.26)$ & $130.75(21.44)$ \\
\hline 36 M SBP (median, IQR) & $129.00(118.66-141.50)$ & $121.00(118.00-144.00)$ & $131.33(119.75-140.50)$ \\
\hline SBP change (mean, SD) & $-10.45(21.26)$ & $-7.74(23.49)$ & $-11.10(20.98)$ \\
\hline SBP change (median, IQR) & $-9.00(-18.67-1.17)$ & $-6.33(-15.33-0.67)$ & $-9.17(-22.50-1.25)$ \\
\hline \multicolumn{4}{|l|}{ Diabetes measures } \\
\hline Baseline A1c (mean, SD) & $8.42(2.00)$ & $8.28(1.53)$ & $8.45(2.09)$ \\
\hline Baseline A1c (median, & $8.10(6.90-9.75)$ & $8.20(7.05-8.95)$ & $8.10(6.70-9.80)$ \\
\hline \multicolumn{4}{|l|}{ IQR) } \\
\hline \multicolumn{4}{|l|}{ Kidney function measures } \\
\hline Baseline eGFR (mean, SD) & $80.93(21.03)$ & $78.35(18.52)$ & $81.46(21.46)$ \\
\hline Baseline eGFR (median, & $85.53(64.74-94.05)$ & $81.46(68.57-91.71)$ & $85.76(64.01-97.33)$ \\
\hline \multicolumn{4}{|l|}{ IQR) } \\
\hline \multicolumn{4}{|l|}{ Comorbidities $(N, \%)$} \\
\hline Chronic kidney disease $^{\dagger}$ & $5(8.47)$ & $0(0.00)$ & $5(10.20)$ \\
\hline Hypertension ${ }^{\dagger}$ & $56(94.92)$ & $10(100.00)$ & $46(93.88)$ \\
\hline High cholesterol $^{\dagger}$ & $41(69.49)$ & $7(70.00)$ & $34(69.39)$ \\
\hline Cardiovascular disease & $8(13.56)$ & $1(10.00)$ & $7(14.29)$ \\
\hline \multicolumn{4}{|l|}{ Pharmacist recommendations } \\
\hline Recommendations (mean, & $2.98(2.31)$ & $2.20(1.40)$ & $3.14(2.43)$ \\
\hline SD) & & & \\
\hline Recommendations (median, & $2.00(1.00-4.00)$ & $1.50(1.00-3.75)$ & $2.00(1.00-5.00)$ \\
\hline \multicolumn{4}{|l|}{ IQR) } \\
\hline \multicolumn{4}{|l|}{ Recommendations $(N, \%)$} \\
\hline 1 recommendation & $19(32.20)$ & $5(50.00)$ & $14(28.57)$ \\
\hline 2 recommendations & $12(20.34)$ & $1(10.00)$ & $11(22.45)$ \\
\hline 3 recommendations & $10(16.95)$ & $1(10.00)$ & $9(18.37)$ \\
\hline$\geq 4$ recommendations & $18(30.51)$ & $3(30.00)$ & $15(30.61)$ \\
\hline Accepted by PCP (mean, & $1.81(1.51)$ & $0.00(0.00)$ & $2.18(1.39)$ \\
\hline \multicolumn{4}{|l|}{ SD) } \\
\hline Accepted by PCP (median, & $1.00(1.00-3.00)$ & $0.00(0.00-0.00)$ & $2.00(1.00-3.00)$ \\
\hline IQR) & & & \\
\hline \multicolumn{4}{|l|}{ Accepted by PCP $(N, \%)$} \\
\hline 0 recommendations & $10(16.95)$ & $10(100.00)$ & $0(0.00)$ \\
\hline 1 recommendation & $22(37.29)$ & $0(0.00)$ & $22(44.90)$ \\
\hline 2 recommendations & $10(16.95)$ & $0(0.00)$ & $10(20.41)$ \\
\hline 3 recommendations & $9(15.25)$ & $0(0.00)$ & $9(18.37)$ \\
\hline$\geq 4$ recommendations & $8(13.56)$ & $0(0.00)$ & $8(16.33)$ \\
\hline
\end{tabular}

36 M 36 months, eGFR estimated glomerular filtration rate, IQR interquartile range, PCP primary care provider, SBP systolic blood pressure, SD standard deviation

*Change in baseline and 36-month systolic blood pressure was calculated for study participants with valid measures (47 total participants, 9 participants with no recommendations accepted by a PCP, and 38 participants with at least 1 recommendation accepted by a PCP)

†Chronic kidney disease, hypertension, and high cholesterol were self-reported by participants. Participants responded to questions regarding ever being told by a doctor or other health professional that they had these conditions

+ Cardiovascular disease included electronic medical record history of at least one myocardial infarction, congestive heart failure, or stroke

management and medication needs. The STOP-DKD study incorporated innovative team-based care models (e.g., support from pharmacists and PCPs) as well as non-traditional modes of delivery (e.g., telephone-based care).
Our analysis demonstrates that a clinically relevant group of participants $(43 \%)$ had at least one medication-related recommendation and they were accepted over half of the time $(61 \%)$. This suggests that pharmacists may identify important unmet 
Table 3 Associations Between Pharmacist Recommendations, Provider Acceptance, and Systolic Blood Pressure

\begin{tabular}{llc}
\hline \hline Variables & $\begin{array}{l}\text { Estimate } \\
(\mathbf{9 5 \%} \text { CI) }\end{array}$ & $p$ value \\
\hline $\begin{array}{l}\text { GLMM } \\
\quad \text { Number of recommendations }\end{array}$ & $1.09(1.00,1.42)$ & 0.0496 \\
$\begin{array}{l}\text { LR model 1 } \\
\quad \text { Number of recommendations }\end{array}$ & $-0.21(-3.72,3.30)$ & 0.9073 \\
$\quad \begin{array}{l}\text { Number of accepted } \\
\text { recommendations }\end{array}$ & $-2.43(-7.77,2.90)$ & 0.3759 \\
$\quad \begin{array}{l}\text { Baseline SBP } \\
\quad \text { mercent of accepted } \\
\text { recommendations }\end{array}$ & $0.45(0.03,0.88)$ & 0.0439 \\
$\quad$ Baseline SBP & $2.36(-12.93,17.63)$ & 0.7640 \\
\hline $\begin{array}{l}\text { CI confidence interval, GLMM generalized linear mixed model, LR } \\
\text { linear regression, SBP systolic blood pressure }\end{array}$ & \\
$*$ GLMM reports odds ratios and LR models report parameter estimates
\end{tabular}

clinical needs and provide actionable suggestions for PCPs. In an era where primary care is stretched thin, this two-pronged approach of engaging pharmacists and using telephone-based interventions may facilitate the reduction of unnecessary inperson primary care visits while simultaneously helping PCPs focus their energy on complex cases, such as patients experiencing uncontrolled comorbidities or medication intolerance.

Additionally, we noted that the number of pharmacist recommendations was marginally correlated with PCP acceptance. This suggests that there may be a saturation point at which recommendations become diluted and no longer actionable. Future research is needed to identify this threshold, noting that there could be different thresholds for different conditions and at different points along the treatment trajectory. Finding this "sweet spot" has the potential to not only reduce clinical inertia, but also maximize the impact of pharmacist-directed care. The quality of pharmacists'

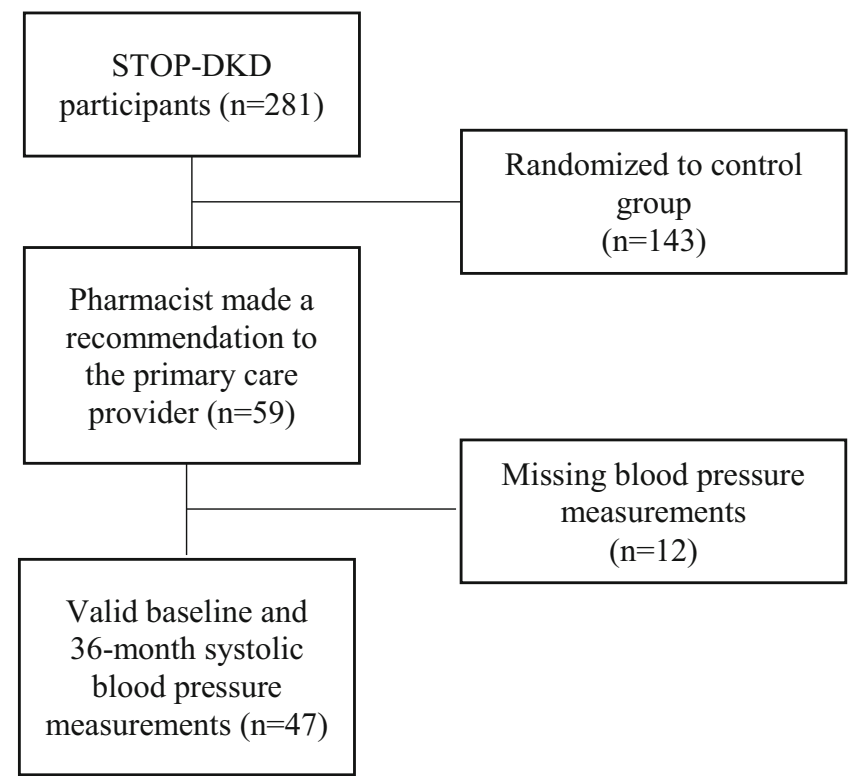

Figure 1 STOP-DKD participant flow. recommendations was likely a critical component of whether physicians accepted recommendations; however, a formal assessment of quality was not part of this study. There were instances when a pharmacist made the same recommendation for a participant over time and it was accepted the second time, suggesting that elements of recommendation quality, repetition, approval, and other factors could be in play. In addition, the impact of pharmacists' recommendations on blood pressure control was potentially limited because PCP approval was required; pharmacists were not directly able to implement their own suggestions. Finally, pharmacists had access to patients' home-monitored blood pressure values, but PCPs did not necessarily have the ability to view this data on which pharmacist recommendations were based.

We also observed a significant decline in SBP at 36 months, but found no evidence that this reduction was associated with pharmacist recommendations in our limited sample. Ongoing debate surrounding SBP targets ${ }^{2}$ (and the observational studies on which some guidelines are based $)^{6}$ may have prevented PCPs from accepting treatment recommendations, especially for patients with high baseline blood pressure. Clinical evidence has demonstrated that intensive blood pressure control is not associated with a reduced risk of kidney failure and, in some cases, may increase the risk of impaired kidney function, congestive heart failure events, and all-cause mortality. ${ }^{2,3,6}$ Even in cases where PCPs accepted pharmacist recommendations, patient factors may have influenced SBP at 36 months. Patients with high baseline blood pressure may have experienced common barriers to medication adherence (e.g., cost of and inability to pay for medication) $)^{21}$ that may have led to uncontrolled SBP at follow-up. Of note, the pathophysiology of hypertension in DKD is complex, primarily due to the negative influence of kidney dysfunction on blood pressure control. This makes hypertension in DKD distinct from essential hypertension alone. As such, primary therapies for hypertension in DKD typically involve prioritization of reninangiotensin-aldosterone system (RAAS) inhibition and albuminuria reduction. Similarly, blood pressure targets in patients with DKD are lower than for those without diabetes to minimize the synergistic influence of hypertension, diabetes, and kidney disease. ${ }^{22}$

Our study had several limitations, chiefly a small sample size and inability to adjust for potential confounders, which limit the interpretation and generalizability of our findings. Future studies would ideally include a larger number of pharmacists providing recommendations and subsequent recommendations, which could enable a robust evaluation of the quality of recommendations. Due to a small sample, models were not adjusted for potential measured confounders (e.g., age, sex, comorbidities, change in underlying A1c control, and/or diabetes progression). In addition, there were several possible confounders that we were unable to assess, including a PCP effect, whereby individual providers or providers with certain characteristics may be more or less likely to accept 
pharmacists' recommendations. Similarly, there could be a pharmacist effect where different pharmacists might have different patterns of recommendations (e.g., number or content of recommendations) that could impact a provider's likelihood of acceptance. It is worth noting that medication adherence is influenced by multiple factors at the patient, provider, and healthcare system levels, and there is a complex interaction between these factors. For example, a patient's beliefs about medications can be influenced by a provider's communication style, which in turn might be impacted by a healthcare system's focus on medication adherence-related quality measures. Lastly, there could be a recommendation effect. For example, simpler, less intense recommendations (e.g., reducing a dose, removing a medication) might be perceived as easier or safer to implement. These are all potential avenues for future research.

Despite these limitations, our analysis has several important clinical implications. First, team-based care is an important model with potential to improve blood pressure control among patients with complex chronic conditions like DKD. Second, our work demonstrates that pharmacists can provide actionable medication-related recommendations and that PCPs are generally accepting of such recommendations. This approach could be used to complement comprehensive management programs, which are increasingly viewed as a best practice for managing multiple chronic conditions. ${ }^{23-26}$ Finally, including telephone-based care provided by pharmacists may have important implications for potentially reducing primary care visits and ensuring the best possible care in between traditional office visits.

Corresponding Author: Hayden B. Bosworth, PhD; Center of Innovation to Accelerate Discovery and Practice Transformation (ADAPT), Durham Veterans Affairs Health Care System, Durham, NC, USA (e-mail: boswo001@duke.edu).

Funding Information The research reported in this publication was supported by the National Institute of Diabetes and Digestive and Kidney Diseases of the National Institutes of Health (NIH) under award no. 1R01DK093938. Dr. Zullig was supported by a VA Health Service Research and Development (HSR\&D) Career Development Award (CDA 13-025). Dr. Davenport was partially supported by the NIH Clinical and Translational Science Award at Duke (UL1TRO02553). Dr. Diamantidis was supported by grants funded by the National Institute of Diabetes and Digestive and Kidney Diseases (K23-DK099385 \& R01-DK093938). Dr. Bosworth was supported by a Research Career Scientist award from VA HSR\&D (VA HSR\&D 08-027). The authors thank the Center of Innovation to Accelerate Discovery and Practice Transformation (ADAPT) at the Durham Veterans Affairs Health Care System for their support.

\section{Compliance with ethical standards:}

Conflict of Interest: Dr. Zullig reports research grant support from the PhRMA Foundation, Proteus Digital Health and Sanofi, as well as consulting from Novartis. Dr. Davenport, Dr. Diamantidis, Dr. Moaddeb, Ms. Jazowski, Ms. Patel, and Ms. Oakes report no conflicts of interest. Dr. Bosworth reports research grants from Sanofi, PhRMA Foundation, Proteus Digital Health, Otsuka, Novo Nordisk, Improved Patient Outcomes, as well as consulting from Novartis, Otsuka, Abbott, and Sanofi.
Disclaimer: The content is solely the responsibility of the authors and does not necessarily reflect the position or policy of Duke University, the US Department of Veterans Affairs, or the US government.

\section{REFERENCES}

1. Thorton Snider J, Sullivan J, van Eijndhoven E, et al. Lifetime benefits of early detection of diabetic kidney disease. PLoS One. 2019;14(5):e0217487.

2. Thomas MC, Brownlee M, Susztak K, et al. Diabetic kidney disease. Nat Rev Dis Primers. 2015;1:15018.

3. Alicic RZ, Rooney MT, Tuttle KR. Diabetic kidney disease: challenges, progress, and possibilities. Clin J Am Soc Nephrol. 2017;12(12):20322045.

4. Helou N, Talhouedec D, Shaha M, Zanchi A. The impact of a multidisciplinary self-care management program on quality of life, self-care, adherence to anti-hypertensive therapy, glycemic control, and renal function in diabetic kidney disease: a cross-over study protocol. BMC Nephrol. 2016;17:88.

5. Patney V, Whaley-Connell A, Bakris G. Hypertension management in diabetic kidney disease. Diabetes Spectr. 2015;28(3):175-180.

6. Doshi SM, Friedman AN. Diagnosis and management of type 2 diabetic kidney disease. Clin J Am Soc Nephrol. 2017;x12(8):1366-1373.

7. Li T, Wu HM, Wang F, et al. Education programmes for people with diabetic kidney disease. Cochroan Database Syst Rev. 2011;6:CD007374.

8. Leung WY, So WY, Tong PC, Chan NN, Chan JC. Effects of structured care by a pharmacist-diabetes specialist team in patients with type 2 diabetic nephropathy. Am J Med. 2005;118(12): 1414.

9. Conn VS, Ruppar TM. Medication adherence outcomes of 771 intervention trials: systematic review and meta-analysis. Prev Med. 2017;99:269276.

10. Fazel MT, Bagalagel A, Lee JK, Martin JR, Slack MK. Impact of diabetes care by pharmacists as part of health care team in ambulatory settings: a systematic review and meta-analysis. Ann Pharmacother. 2017;51(10):890-907.

11. Pape GA, Hunt JS, Butler KL, et al. Team-based care approach to cholesterol management in diabetes mellitus: two-year cluster randomized controlled trial. Arch Intern Med. 2011;171(16):1480-1486.

12. Niznik JD, He H, Kane-Gill SL. Impact of clinical pharmacist services delivered via telemedicine in the outpatient or ambulatory care setting: a systematic review. Res Social Adm Pharm. 2018;14(8):707-717.

13. Abughosh SM, Wang X, Serna O, et al. A pharmacist telephone intervention to identify adherence barriers and improve adherence among nonadherent patients with comorbid hypertension and diabetes in a medicare advantage plan. J Manag Care Spec Pharm. 2016;22(1):6373.

14. Lyons I, Barber N, Raynor DK, Wei L. The medicines advice service evaluation (MASE): a randomised controlled trial of a pharmacist-led telephone based intervention designed to improve medication adherence. BMJ Qual Saf. 2016;25(10):759-769.

15. Scala D, Menditto E, Caruso G, et al. Are you more concerned about or relieved by medicines? An explorative randomized study of the impact of telephone counseling by pharmacists on patients' beliefs regarding medicines and blood pressure control. Patient Educ Couns. 2018;101(4):679-686.

16. Singleton J, Veach S, Catney C, Witry M. Analysis of a community pharmacy intervention to improve low adherence rates to oral diabetes medications. Pharmacy (Basel). 2017;5(4):E58

17. Diamantidis CJ, Bosworth HB, Oakes MM, et al. Simultaneous risk factor control using telehealth to slow progression of diabetic kidney disease (STOP-DKD) study: protocol and baseline characteristics of a randomized controlled trial. Contemp Clin Trials. 2018;69:28-39.

18. Zullig LL, Diamantidis CJ, Bosworth HB, et al. Racial differences in nocturnal dipping status in diabetic kidney disease: results from the STOP-DKD (simultaneous risk factor control using telehealth to slow progression of diabetic kidney disease) study. J Clin Hypertens (Greenwich). 2017;19(12):1327-1335.

19. de Boer IH, Rue TC, Hall YN, Heagerty PJ, Weiss, NS, Himmelfarb J. Temporal trends in the prevalence of diabetic kidney disease in the United States. JAMA. 2011;305(24):2532-2539.

20. Duru OK, Middleton T, Tewari MK, Norris K. The landscape of diabetic kidney disease in the United States. Curr Diab Rep. 2018;18(3): 14. 
21. Williams AF, Manias E, Walker R. Adherence to multiple, prescribed medications in diabetic kidney disease: a qualitative study of consumers' and health professionals' perspectives. Int J Nurs Stud. 2008;45(12): 1742-1756.

22. Van Buren PN, Toto R. Hypertension in diabetic nephropathy: epidemiology, mechanisms, and management. Adv Chronic Kidney Dis. 2011;18(1):28-41.

23. de Bruin SR, Versnel N, Lemmens LC, et al. Comprehensive care programs for patients with multiple chronic conditions: a systematic literature review. Health Policy. 2012;107(2-3):108-145.

24. Hopman P, de Bruin SR, Forjaz MJ, et al. Effectiveness of comprehensive care programs for patients with multiple chronic conditions or frailty: a systematic literature review. Health Policy. 2016;120(7):818-832.
25. Bleich SN, Sherrod C, Chiang A, et al. Systematic review of programs treating high-need and high-cost people with multiple chronic diseases or disabilities in the United States, 2008-2014. Prev Chronic Dis. 2015;12:E197.

26. Smith SM, Wallace E, O'Dowd T, Fortin M. Interventions for improving outcomes in patients with multimorbidity in primary care and community settings. Cochrane Database Syst Rev. 2016;3:CD006560.

Publisher's Note Springer Nature remains neutral with regard to jurisdictional claims in published maps and institutional affiliations. 